\title{
Bilirrubina: um Novo Marcador na Doença Arterial Coronariana?
} Ver artigo relacionado
na página 445

\author{
Carísi A. Polanczyk ${ }^{1}$
}

I mportantes avanços foram feitos nos últimos anos na identificação de novos fatores relacionados a maior risco ou probabilidade de desenvolver doença aterosclerótica coronariana. Além dos fatores de risco tradicionais, recentemente agregamos informações sobre marcadores inflamatórios, como a proteína C-reativa, e marcadores de lesão miocárdica, como as troponinas, e também de alteração hemodinâmica, como o peptídeo atrial natriurético (BNP). Cada um, dentro de um contexto, tem demonstrado ser útil ao fornecer informações prognósticas e guiar condutas terapêuticas. Por outro lado, ainda estamos muito distantes do cenário ideal. Não conseguimos demonstrar que os imunossupressores podem reduzir a doença aterosclerótica ou mesmo usar marcadores existentes para identificar precocemente casos com doença subclínica, silenciosa ou com risco iminente de instabilização. Precisamos aprofundar o entendimento pleno da fisiopatogenia da doença arterial coronariana (DAC), suas causas e fatores de risco.

Nesse sentido, é importante o estudo de Ghem et al. ${ }^{1}$, publicado neste fascículo da Revista Brasileira de Cardiologia Invasiva, sobre o valor de um novo preditor como agravante da DAC. A bilirrubina vem sendo considerada na patogênese da DAC, após estudos observacionais do início da década de 90 demonstrarem que níveis séricos de bilirrubina estavam inversamente relacionados com presença de DAC. Schwertner et al. ${ }^{2}$ demonstraram, em uma amostra de 619 homens, que redução de 50\% na bilirrubina total estava associada a aumento de $47 \%$ nas chances de DAC. Níveis elevados de colesterol de lipoproteína de baixa densidade (LDL-colesterol) e sua modificação oxidativa têm sido relacionados com esse processo ${ }^{3}$. A bilirrubina, um antioxidante, parece prevenir a modificação do LDL e poderia proteger contra a aterogênese. Estudos em indivíduos normais e com doença aterosclerótica demonstraram relação inversa entre níveis de bilirrubina sérica, dentro de níveis de normalidade, com disfunção endotelial e espessamento da camada íntima-média carotídea ${ }^{4}$. Além da ação antioxidante, a bilirrubina tem sido relacionada com inibição da expressão de moléculas de adesão e proliferação celular ${ }^{5}$. Por outro lado, apesar desses achados, outros autores não conseguiram identificar essa relação entre bilirrubina e níveis de ferro com doença aterosclerótica ${ }^{6}$.

No estudo de Ghem et al. ${ }^{1}, 81$ pacientes com DAC foram analisados e os níveis séricos de bilirrubina foram avaliados e relacionados com a carga aterosclerótica em artérias coronárias, por meio do escore validado de Humphries. Os resultados demonstraram ausência de associação entre níveis séricos de bilirrubina e gravidade da doença coronariana. Diante desses achados, é oportuno discutirmos se existem realmente evidências de que a bilirrubina possa estar relacionada de modo independente com a gênese da doença aterosclerótica ou as evidências ainda são fracas, com potenciais vieses que turvam uma análise adequada. Nesse sentido, cabem algumas considerações metodológicas em relação ao estudo. Conforme apontado pelos autores, o tamanho da amostra é limitado e a falta de associação não permite refutar a hipótese com certeza. Por exemplo, é conhecido que níveis de LDL-colesterol e de colesterol de lipoproteína da alta densidade (HDL-colesterol), bem como a proteína C-reativa, estão relacionados com a extensão e a gravidade da doença aterosclerótica. Nesse estudo de Ghem et al. ${ }^{1}$, esses e outros fatores de risco não foram diferentes entre o grupo com doença leve/moderada ou grave. Além disso, é possível que se trate de uma amostra de indivíduos em tratamento para doença coronariana, com provável uso de estatinas e antiagregantes plaquetários. A enzima heme-oxi-

\footnotetext{
1 Serviço de Cardiologia do Hospital de Clínicas de Porto Alegre, Programa de Pós-graduação em Medicina: Cardiologia e Epidemiologia, Faculdade de Medicina, Universidade Federal do Rio Grande do Sul - Porto Alegre, RS.

Correspondência: Carísi A. Polanczyk. Serviço de Cardiologia. Hospital de Clínicas de Porto Alegre. Rua Ramiro Barcelos, 2.350 - sala 2.225

- Porto Alegre, RS - CEP 90035-003

E-mail: cpolanczyk@hcpa.ufrgs.br

Recebido em: 5/12/2008 • Aceito em: 8/12/2008
} 
genase-1 (HO-1), por exemplo, tem papel central nas ações antioxidantes e antiinflamatórias, que podem ser mediadas pela formação de biliverdina/bilirrubina e monóxido de carbono. Inibidores da HMG-CoA redutase (estatinas) induzem expressão in vitro de HO-1 e têm sido relacionados com uma pletora de efeitos antioxidativos nos vasos ${ }^{7}$. Apesar de os autores não terem relatado uso de medicações, o emprego desses fármacos poderia interferir na associação entre níveis de bilirrubina e ferro e extensão da doença no momento avaliado.

É importante não perdermos a perspectiva de que muito ainda precisa ser esclarecido em relação aos mecanismos celulares, genéticos e moleculares relacionados com a doença aterosclerótica. Novas hipóteses e novos marcadores precisam ser avaliados e reavaliados para avançarmos no conhecimento. Não podemos nos conformar com o conhecimento existente ou diante de estudos inicialmente negativos. Cabe à comunidade científica um olhar crítico e ambicioso para continuar desbravando essa doença, que, apesar de todos os avanços, continua liderando a posição de principal causa de morte em nosso País.

\section{REFERÊNCIAS BIBLIOGRÁFICAS}

1. Ghem C, Sarmento-Leite R, Quadros AS, Rossetto S, Gottschall CAM. Associação da concentração de bilirrubina sérica com a carga aterosclerótica em pacientes com doença arterial coronariana estabelecida. Rev Bras Cardiol Invas. 2008;16(4):445-9.

2. Schwertner HA, Jackson WG, Tolan G. Association of low serum concentration of bilirubin with increased risk of coronary artery disease. Clin Chem. 1994;40(1):18-23.

3. Chen YH, Chau LY, Chen JW, Lin SJ. Serum bilirubin and ferritin levels link heme oxygenase-1 gene promoter polymorphism and susceptibility to coronary artery disease in diabetic patients. Diabetes Care. 2008;31(8):1615-20.

4. Erdogan D, Gullu H, Yildirim E, Tok D, Kirbas I, Ciftci O, et al. Low serum bilirubin levels are independently and inversely related to impaired flow-mediated vasodilation and increased carotid intima-media thickness in both men and women. Atherosclerosis. 2006;184(2):431-7.

5. Ollinger R, Bilban M, Erat A, Froio A, McDaid J, Tyagi S, et al. Bilirubin: a natural inhibitor of vascular smooth muscle cell proliferation. Circulation. 2005;112(7):1030-9.

6. Danesh J, Appleby P. Coronary heart disease and iron status: meta-analyses of prospective studies. Circulation. 1999;99(7): 852-4.

7. Hsu M, Muchova L, Morioka I, Wong RJ, Schröder H, Stevenson DK. Tissue-specific effects of statins on the expression of heme oxygenase- 1 in vivo. Biochem Biophys Res Commun. 2006;343(3):738-44. 